\title{
Influencia de la sintaxis del español en el aprendizaje del inglés
}

Enviado: 30 de septiembre de 2020/ Aceptado: 3 de marzo de 2021 / Publicado: 12 de julio de 2021

AMAURY BELTRÉ-GARCÍA

Departamento de Lenguas Extranjeras, Instituto Superior de Formación Docente Salomé Ureña,

República Dominicana

amaury.beltre@isfodosu.edu.do

\section{RESUMEN}

El estudio de las formas en las que el español como lengua materna influye en el aprendizaje de inglés como lengua extranjera es un tema de interés para la comunidad científica y docente (Matias, 2019; Relyea y Amendum, 2019; Rodríguez, 2018). En ese sentido, este artículo tiene por finalidad describir cómo la sintaxis del español (L1) influye en el aprendizaje del inglés como lengua extranjera. Para cumplir con dicho objetivo se les pidió a 14 estudiantes completar un preexamen, componer un texto narrativo, tomar un examen de nivel, y responder a una encuesta sobre la frecuencia con la que refuerzan fuera de clases los contenidos que aprenden en el aula. Y a la maestra se le realizó una entrevista en profundidad. Los resultados se analizaron partiendo de un enfoque cualitativo con ayuda de técnicas de análisis cuantitativas. Los hallazgos señalan que el español influye negativamente en el aprendizaje de inglés debido a la traducción literal y a la poca compresión de la gramática del inglés, además de las similitudes lingüísticas existentes entre los dos idiomas que causan que

\section{ABSTRACT}

Influence of Spanish Syntax on the Learning of English

The study of the ways in which Spanish as a mother tongue influences the learning of English as a foreign language is a topic of interest for the scientific and teaching community (Matias, 2019; Relyea and Amendum, 2019; Rodríguez, 2018). In that sense, this article aims to describe how the syntax of Spanish (L1) influences the learning of English as a foreign language. To fulfill this objective, 14 students were asked to complete a pre-test, compose a narrative text, take a placement test, and respond to a survey on how often they reinforce outside of class the content they learn in the classroom, and the teacher was given an in-depth interview. The results were analyzed using a qualitative approach with the help of quantitative analysis techniques. The findings indicate that Spanish has a negative influence on English learning due to literal translation and poor understanding of English grammar, in addition to the linguistic similarities between the two languages that 
los estudiantes intercambien de forma deliberada la gramática de su lengua materna con la del inglés.

Palabras Clave: lengua materna, segunda lengua, interlenguaje, transferencia lingüística, traducción literal. cause students to deliberately exchange their native language grammar with English.

Keywords: mother tongue, second language, interlanguage, language transfer, literal translation.

\section{INTRODUCCIÓN}

La necesidad principal en el uso de lenguas humanas no es biológica, tampoco es de supervivencia y/o la fecundidad, pero sí de suficiente producción expresiva para suplir las necesidades de la comunidad, la maximización del éxito comunicativo y la reducción del esfuerzo cognitivo (Steels, 2017). De acuerdo con Mueller (2018) el inglés es considerado como lingua franca ya que se utiliza como idioma oficial en más de 70 países, y es considerado el idioma extranjero más enseñado en más de 100 países de todo el mundo. Este estatus de idioma global explica porque el aprendizaje de inglés ha tomado gran fuerza en latino-américa en los últimos años (Burgos y Molina 2020).

En esa misma tesitura, el cúmulo de estudios sobre la adquisición de una segunda lengua hechos por especialistas en el área (Kormos, 2020; VanPatten, Keating, y Wulff, 2020) han generado múltiples debates sobre las diferentes formas para propiciar el aprendizaje de inglés de acuerdo con los contextos en los que se enseña, las dificultades que presentan los estudiantes, y las diversas teorías existentes sobre este tema. En ese sentido, Matias (2019); Relyea y Amendum (2019); Rodríguez (2018); Kelley, Roe, Blanchard y Atwill (2015) estudiaron los diferentes aspectos en los cuales español como lengua materna (L1) puede influir en el aprendizaje de inglés como lengua extranjera. Estos autores y la búsqueda de evidencias que expliquen este fenómeno condujeron a observaciones minuciosas en las que se pudo notar que muchos estudiantes de EFL estructuran sus ideas en inglés de manera similar a como lo hacen en español.

En ese aspecto, esta investigación es motivada por cuatro objetivos: 1) describir las estructuras sintácticas del español que influyen en el aprendizaje del inglés; 2) determinar la relación del nivel de inglés de los estudiantes con el nivel del programa básico (A2) del instituto Crece; 3) identificar la frecuencia con que los estudiantes refuerzan fuera de clases los contenidos aprendidos en el aula, e 4) interpretar la percepción de la maestra de inglés con relación a las estructuras sintácticas del español que influyen en el aprendizaje del inglés. 


\section{MARCO TEÓRICO}

Los estudiantes de EFL principiantes dependen básicamente de su lengua materna (Eddin y Hamza, 2019), esto los lleva a cometer diferentes tipos de errores que reflejan la adaptación de recursos lingüísticos de la lengua materna a las propiedades ortográficas de la segunda lengua Ellis (1994 citado en Li 2019; Zaman, Qi, Rafique, Fazal, Asif, y Shan; 2019; Lallier y Carreiras, 2018). Esta influencia lingüística puede ser fonológica, morfológica, sintáctica y semántica (Lao, 2017).

El análisis de la influencia de una lengua sobre otra está sujeto a las teorías del interlanguage, the significance of learner's error y del constrative analysis. Singh, Singh, Razak, y Ravinthar (2017) y Khansir (2012) explican que error-análisis es un tipo de análisis lingüístico centrado en comparar los errores gramaticales que cometen los estudiantes en la segunda lengua con la gramática de la lengua materna. Mediante este análisis profundo los lingüistas, investigadores y docentes pueden comprender con más claridad el proceso de aprendizaje en ambas lenguas.

Sin embargo, comparar la gramática del L1 y con la de lengua extranjera no es suficiente para determinar si el estudiante ha cometido un error, para identificar la presencia y la naturaleza de un error es necesario hacer una interpretación de la expresión del estudiante (Filipovic, 2018), ya que este prodría usar su conocimiento cultural de la lengua materna para expresar significando en la segunda lengua. En consonancia con lo mencionado, Sharma y Tripathi (2018); Shum, Ho, Siegel, y Au (2016) explican que se pueden predecir los elementos de la segunda lengua que serán difíciles de aprender para los estudiantes, compararando sistemáticamente ambos idiomas (L1 y L2) y sus culturas. Cuando los dos idiomas y culturas son similares no habrá dificultades de aprendizaje, pero cuando son diferentes es de esperarse que surjan dificultades: a mayor diferencia entre la lengua materna y su cultura en relación con la lengua extranjera, mayor grado de dificultad en el aprendizaje de la segunda (Choi, 2017 citando a Lado, 1957).

\subsection{Interlenguaje}

Richards et al (1996 citado por Al-Khresheh, 2015) postulan que los estudiantes en proceso de aprender una segunda lengua producen un tipo de lenguaje independiente a la lengua materna y a la segunda lengua. Este es un sistema intermedio entre ambas lenguas, que incorpora elementos del L1 y de la segunda lengua (Benati, 2017 citando a Selinker, 1972; Brown, 2014). 
Los interlenguajes son lenguas intermedias que se crean cuando un estudiante intenta crear y expresar un significado en una situación de aprendizaje significativo en su segunda lengua (Selinker, 1992 citado en Yang y Xu, 2019). Este se ve afectado por la lengua materna, ya que el estudiante utiliza su conocimiento del L1 para entender y organizar sus ideas en la segunda lengua y/o para compensar la falta de competencias lingüísticas existentes en la lengua objeto (Cervatiuc, 2019).

En ese mismo tenor, se entiende que el interlenguaje es sistemático en todo su desarrollo (Tarone, 2006) el mismo refleja los intentos del estudiante en construir un sistema lingüístico que se parezca al idioma que aprende (Corder, 1978 citado en Moeller y Catalano (2015), y tiene todas las propiedades normales de los lenguajes naturales: se desarrolla de manera sistemática, se desarrolla con el tiempo de acuerdo con la cantidad de input de la segunda lengua que reciba el estudiante y está sujeto a normas de la misma manera que cualquier otra lengua (Fauziati y Maftuhin, 2016 citando a Selinker, 1997; Tarone, 2006).

El interlenguaje se basa en la sobre-generalización a partir de patrones encontrados en el idioma que se aprende, la transferencia a partir de patrones encontrados en el idioma nativo del estudiante, y la fosilización, el fenómeno en el cual el idioma del estudiante deja de desarrollarse (Han y Tarone, 2014; Larsen-Freeman, 2014).

\subsection{Sobre-Generalización}

Los estudiantes asumen que muchas de las reglas y formas en la segunda lengua son similares a las de su lengua nativa (Kalee, Rasyid y Muliastuti, 2018 citando a Richards, 1974; Brown, 2014), y las aplican en contextos o casos en los que tales reglas no son de uso pertinente (Mahmood y Murad, 2018).

\subsection{Transferencia Lingüística}

La transferencia lingüística es la distribución de formas y significados desde la lengua y cultura nativa a la lengua y cultura extranjera (Lado, 1957 citado en Khalifa, 2018; Rodríguez, 2018). La transferencia lingüística es bidireccional (Albirini y Benmamoun, 2014). La forma en que los estudiantes organizan las ideas en su lengua materna influye en la transferibilidad a la segunda lengua (Huang, 2017). Mientras mejor establecida esté la lengua materna al momento del aprendizaje del L2, mayor será su influencia en la segunda lengua (Guion, Flege, Liu, y Yeni-Komshian, 2000). El nivel de competencias que los estudiantes desarrollen en la segunda

4 International Journal of New Education | Núm. 7 
lengua va a estar significativamente influenciado por las competencias que hayan desarrollado en su L1 (Cummins, 1979 citado por Mohammad y Taie, 2016).

Transferencia positiva. sucede cuando las estructuras que el estudiante transfiere desde la lengua materna a la segunda lengua son equivalentes (Littlewood, 1984 citado por Khalifa, 2018). Es decir, las estructuras trasnferidas desde la L1 facilitan el aprendizaje de la segunda lengua (Li, 2019).

Trasferencia negativa. es cuando las estructuras de la lengua materna que el estudiante transfiere no son equivalentes a las de la segunda lengua (Broselow, 1984 citado en Khalifa, 2018). Esta se debe principalmente a las diferencias gramaticales entre ambas lenguas que los estudiantes intercambian de manera deliberada (Li, 2019).

\subsection{Fosilización}

En el aprendizaje de segundas lenguas ocurre un fenómeno psicolingüístico manifestado como elementos, reglas y subsistemas lingüísticos que los estudiantes tienden a mantener en su interlenguaje independientemente de la edad o de la cantidad de explicación e instrucción que reciba en la segunda lengua (Han, 2012 citando a Selinker, 1972; Abbas, Younus y Khalil, 2019; Yang y Xu, 2019).

\section{MÉTODO}

Esta investigación está enmarcada dentro de las investigaciones mixtas. Creswell (2003, citado en Techo, 2016) y Halcomb y Hickman (2015) explican que un investigador puede optar por emplear un método de investigación mixto en el cual utilice una combinación de métodos cuantitativos y cualitativos para describir completamente un evento, anulando de esta forma los sesgos asociados característicamente con un método de investigación únicamente cualitativo.

En la parte cuantitativa, el investigador hace uso de estadísticos $t$ o t-student (prueba $\mathrm{t}$ pareada) y el alfa de Cronbach y en la parte cualitativa la recolección de datos se hace a través de la aplicación de un examen escrito y de una entrevista en profundidad. 


\subsection{Participantes}

Los participantes de esta investigación conforman un grupo único de 14 estudiantes (nueve hembras y cinco varones) que comprenden edades entre 13 y 17 años, quienes de acuerdo con información suministrada por la maestra, recientemenre iniciaron las clases en nivel A2.

\subsection{Instrumentos}

Para la recolección de datos, el investigador se valió de instrumentos diseñados tomando en cuenta los objetivos de la investigación: A los estudiantes se les aplicó un preexamen de nivel A1; una comnposición en inglés de un texto narrativo sobre su niñez; una encuesta sobre la frecuencia con la que ellos refuerzan fuera de clases los contenidos aprendido en el aula y un examen de nivel A2; y a la maestra una entrevista en profundidad.

\subsection{Procedimientos}

Se pidió a los estudiantes completar un preexamen de nivel (A1) para tener un punto de referencia del nivel con el que ellos entran al curso, después se les solicitó que compusieran un texto narrativo en inglés sobre su infancia, se logró que los estudiantes respondieran a una encuesta sobre la frecuencia con la que ellos refuerzan fuera del aula los contenidos que aprenden en clase. Así mismo, la maestra respondió a una entrevista en profundidad sobre su percepción sobre la influencia del español en el aprendizaje de inglés. Por último, se aplicó un examen de nivel (A2) a los estudiantes para comparar su nivel de entrada y salida del curso.

\section{RESULTADOS}

\subsection{Elementos de las estructuras sintácticas del español que influyen en el aprendizaje del inglés en los estudiantes del nivel básico (A2)}

En las composiciones en inglés hechas por los estudiantes se encontraron casos de transferencia negativa desde el español al inglés como "She asked me why I put this name" en vez de "She asked me why I named him/her/it" esto se debe a que en español comúnmente se dice poner nombres a los bebes, mientras que en inglés seria nombrar los bebes (name the babies). Otro caso de transferencia negativa encontrado fue, "i was running my bike" en lugar de "I was riding my bike" en este caso se puede notar que el escritor usó la minúscula para escribir el pronombre personal (I) ya que en español este pronombre no sigue la regla de las mayúscu- 
las como en inglés. Además, utilizó el verbo (running) que en español es correr para referirse a la acción de montar bicicleta que en inglés es (riding).

Un ejemplo significativo de transferencia negativa es cuando los estudiantes escribieron "when I have 1 year I was very happy" en vez de "When I was 1 year old, I was very happy" esto se presume que sucede debido a que en español se pude utilizar el verbo tener para indicar la edad a diferencia del inglés que se debe indicar con el verbo (to be).

Así mismo se encontró transferencia negativa en el intercambio del pronombre personal en inglés (he) por el articulo definido (the) como sucede en la frase "he dog is very loving" en lugar de "The dog is very loving." Esto sucede porque en español el articulo definido (el) y el pronombre personal (él) se escriben en forma similar. Asi mismo, se puede notar transferencia negativa en la traducion literal del significado de los verbos. Como lo es en caso de "me father wake up the tree" en lugar de "My father lifted the tree" esto se debe a que los verbos (wake up) y (lift) ambos significan levantar en español. Otro ejemplo similar se encuentra en la frase "we put angry" al intentar decir "We got angry" esto ocurre ya que en español se puede decir nos pusimos... para referirse a un estado de ánimo. Ejemplo: Nos pusimos felices de verte, porque nos pusimos triste al saber que partirías hacia tierras lejanas.

En relación a la transferencia negativa desde el español al inglés en el uso del sujeto, se encontró que los estudiantes omiten algunos pronombres personales y/o sustantivos al expresarse en inglés de forma tal como lo harían en español. El análisis mostró que algunos estudiantes escriben "lives in San Juan but before lives in Santo Domingo" la cual es una forma de omisión del sujeto en español, en lugar de "He/she lives in San Juan, but before he/she lived in Santo Domingo" la cual es una forma gramatical perteneciente al inglés. Así mismo se encontró la frase "she is the most, inteligent and beautiful in the world" cuando lo ideal sería "She is the most intelligent and beautiful woman in the world" esto se debe a que el sujeto está relacionado con el género expresado por el adjetivo.

En ese mismo contexto, se puede notar la transferencia negativa desde el español al inglés en la omisión del pronombre objeto en la frase "she wants that will be a good daughter" a causa de que este está contenido en la forma del presente subjuntivo (que yo sea), pero en ingles lo ideal sería "She wants me to be a good daughter."

Otro caso de transferencia negativa se encontró cuando los estudiantes escribieron "I didn't pay attention to she" en lugar de "I didn't pay attention to her" esto se presume ocurre porque (she) y (her) tienen la misma forma de escritura en español (ella). Al igual los pronombres 
anteriores (they) y (them) tienen la misma forma de escritura en español (ellos). Este caso se encontró "them are more older than me" en vez de "They are older than me."

De igual manera, se encnotró transferencia negativa en el uso de cognados. En este caso se nota un nivel de confusión en los estudiantes al usar cognados entre la lengua materna y la lengua extrajera. Los estudiantes escribieron "I did a show and then I calmed down" ya que en español el inglesismo (show) es usado para referirse a cuando una persona se comporta de una manera exagerada. Sin embargo, en inglés la forma ideal de expresar esto sería "I acted a fool, and then I calmed down." De manera similar, se encontró la frase "five nurses having to support me for the doctor can make the cirugy" en la cual la palabra ( $\operatorname{cirug} y)$ no es parte del español, ni tampoco del inglés, y se utilizó el verbo (make) en vez de (perform) ya que ambos verbos en español tienen la misma traducción. La forma ideal de esta frase debe ser "Five nurses had to hold me, so the doctor can perform the surgery."

Dentro de este orden de ideas se encontró la frase "I need to go every Monday for 2 months to inject my ear for total sanation" en la cual la palabra (sanation) no es parte del español, pero tampoco del inglés. Sin embargo, en este caso el termino ideal sería (healing). "I need to visit the doctor's office every Monday for two months to have my ear injected for total healing."

Por otra parte, los estudiantes hicieron transferencia negativa desde el español al inglés al hacer uso inadecuado de los artículos definidos e indefinidos en inglés. Por ejemplo, "he don't like the dogs" utilizando el articulo definido (the) para modificar el sustantivo como sería el caso del español, en lugar de escribir "He doesn't like dogs." Otro ejmeplo, es la frase "she did all the things that the teenagers should to do" en lugar de "She did everything that teenagers should do." En ese mismo tenor se encontró que los estudiantes intercambian el uso de los articulos definidos e indefinido. Por ejemplo, "I get up take the shower" en vez de "I get up and take a shower" y "she doesn't like a dog" en vez de "She doesn't like dogs" utilizanado el artículo indefinido (a) junto a la forma singular del sustantivo ( $\operatorname{dog})$ para referirse a una cantidad en plural.

Volviendo la mirada a la confusión existente en los estudiantes sobre cuando usar cual proposición, se puede apreciar la transferencia negativa desde el español al inglés en el caso de "I drove my bike in the road" en lugar de "I drove my bike on the road" en la cual utilizaron la preposición (in) en vez de (on). De igual manera, "I was in my friend house" en lugar de "I was at my friend's house" se usó (in) en lugar de (at), y "we were playing in the roof of her house" en vez de "we were playing on the roof of her house" usando la preposición (in) en lugar de (on).

Otra forma de transferencia negativa se encontró en el uso de la negación en inglés. Por ejemplo, "my father not like much it" haciendo la negación de manera muy similar a la del español. 
En ese sentido la forma ideal de hacer esta negación en inglés es haciendo uso de (not) más el verbo auxiliar (do) "My father does not like it much." Otros casos encontrados fueron "my sister not found her bag" en lugar de "My sister did not find her bag" y "He said he not see nobody" en lugar de "He said he did not see anybody."

Por último, el uso del adjetivo comparativo y el superlativo al momento de expresarse en inglés muestra que existe una confusión en el uso de ambos resultandos esto en una transferencia negativa. Esto puede ser a razón de que en español el adjetivo comparativo y el superlativo son diferenciado solamente por el uso del artículo definido (el) o (la). Si se traduce la frase escrita por los estudiantes "I wasn't the prettier in the street" del inglés al español, se diría de la siguiente manera "Yo no era la más linda de la calle." En la traducción el adjetivo resulta estar en su forma superlativa. Sin embargo, no es el caso en inglés. La manera adecuada de escribir esta frase en inglés seria "I wasn't the prettiest girl on my street."

\subsection{Relación del nivel de inglés de los estudiantes con el nivel del programa de inglés básico (A2)}

Los resultados de la prueba estadística t-students muestran que lo que se esperaba en cuanto al nivel de conocimiento de los estudiantes al tomar el examen de nivel A2 fue inferior a la esperanza de los resultados obtenidos en el preexamen A1. En ese sentido, las medias fueron 70.4714 para el preexamen A1 y 55.2357 para el examen de nivel A2 lo cual evidencia que existe mucha dispersión ocasionando que la desviación típica sea muy grande, dado que los datos no están correlacionados. Ver figura 1.

Figura 1. Relación preexamen A1 y examen de nivel A2. Fuente: Preexamen A1 y examen de nivel 2A

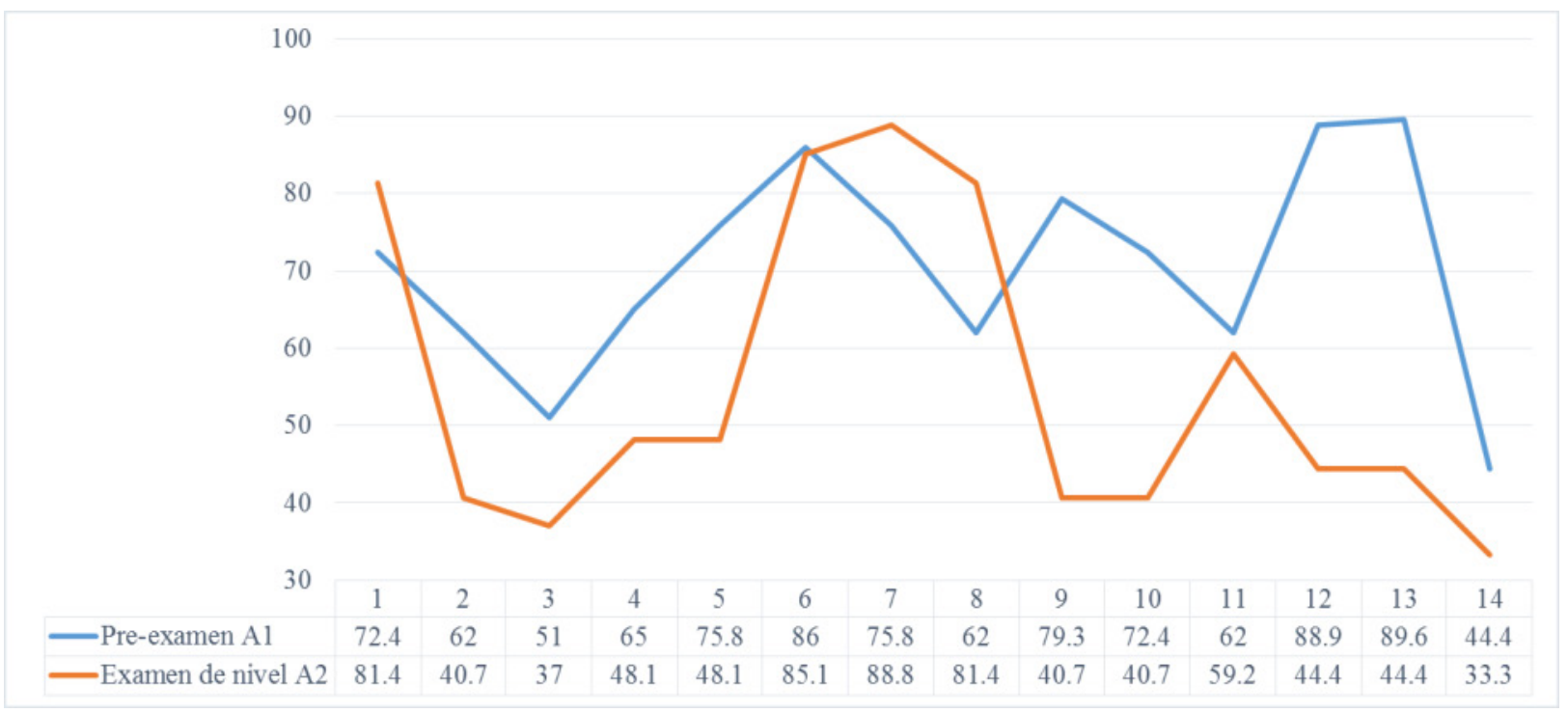


En la curva de los estudiantes 1, 6, 7, y 8 se puede ver un gran desempeño en el examen de nivel A2. El p-valor es 0.017663 el cual indica que ellos lograron adquirir los conocimientos necesarios para aprobar el curso y cumplir con las expectativas. Sin embargo, las curvas de los estudiantes $2,3,4,5$, y 9, 10, 11, 12, 13 muestran que obtuvieron muy bajos conocimientos que no les permitieron aprobar el examen de nivel A2. Al mismo tiempo, la curva atípica del estudiante 14 muestra una puntación muy baja en el examen de nivel A2, lo que indica la falta de conocimientos básicos para aprobar el nivel A2.

\subsection{Frecuencia con la que los estudiantes refuerzan fuera de clases los contenidos aprendidos en el nivel básico (A2)}

Tabla 1. Frecuencia de refuerzo de los contenidos fuera de clases. Fuente: Resultados de la encuesta aplicada a los estudiantes

\begin{tabular}{ll}
\hline Frecuencia & Reforzamiento \\
\hline Todos los días & $29 \%$ \\
\hline Tres veces a la semana & $43 \%$ \\
\hline Una vez a la semana & $21 \%$ \\
\hline Nunca & $7 \%$ \\
\hline
\end{tabular}

Figura 2. Frecuencia de refuerzo de los contenidos fuera de clases. Fuente: Resultados de la encuesta aplicada a los estudiantes

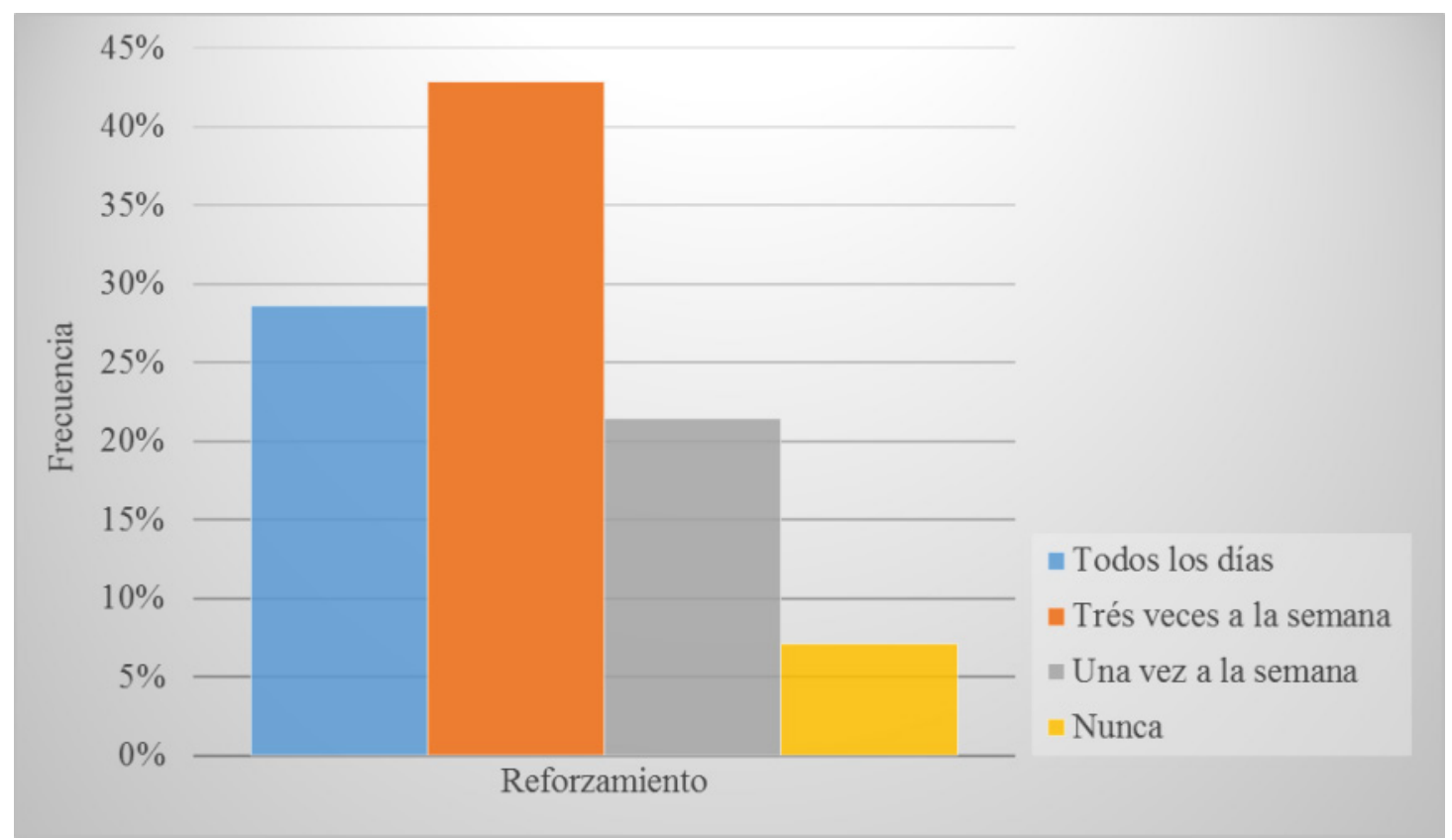


Los resultados de la encuesta aplicada a los estudiantes con el fin de determinar con qué frecuencia ellos refuerzan los contenidos aprendidos fuera del salón de clases muestra que el $29 \%$ de los estudiantes refuerzan los contenidos aprendidos en clases todos los días, un 43\% lo hace tres veces a la semana, un $21 \%$ una vez a la semana y un extraño caso que implica el $7 \%$ de los estudiantes nunca refuerza fuera de clases los contenidos aprendidos en el aula. De estos resultados se puede evidenciar que el $72 \%$ de los estudiantes están dedicando tiempo al estudio individual del idioma inglés, evidenciando su preocupación por aprobar el nivel A2. Ver tabla 1 y figura 2.

Cabe destacar que para determinar el grado de confiabilidad de la encuesta aplicada a los estudiantes se utilizó el alfa de Cronbach, obteniendo un índice de 0.47873597 , lo cual indica que la encuesta tiene una confiabilidad moderada. El resultado del Alfa de Cronbach sugiere que para futuros trabajos es necesario modificar la encuesta con el fin de obtener un grado de confiabilidad más alto.

Tabla 2. Escala de valoración del Alfa de Cronbach. Fuente: Encuesta aplicada a los estudiantes

\begin{tabular}{ll}
\hline Escala & Alfa de Cronbach \\
\hline Alta & $0.8-1.0$ \\
\hline Buena & $0.6-0.8$ \\
\hline Moderada & $0.4-0.6$ \\
\hline Baja & $0.2-0.4$ \\
\hline Muy Baja & $0-0.2$ \\
\hline
\end{tabular}

\subsection{Percepción de la maestra de inglés con relación a las estructuras sintácticas del español que influyen en el aprendizaje del inglés en los estudiantes del nivel básico (A2)}

La entrevista en profundidad realizada a la maestra con el objetivo interpretar su percepción sobre las estructuras sintácticas del español que influyen en el aprendizaje del inglés, arrojó que los estudiantes pricipalamente presentan dificultad en el uso adecuado de los artículos definidos e indefinidos, el manejo de los pronombres personales, el uso de sustantivos y la colocación de los adjetivos.

En ese sentido, la maestra expresó que es muy común que los estudiantes del nivel básico A2 conozcan las reglas de uso de los artículos. Agregando que "we use (the) to refer to specific or particular nouns" o sea, utilizamos 'the' para referirnos a sustantivos particulares o específicos. Sin embargo, en los estudiantes no se percibe un uso fluido al momento de producir de manera independiente, el uso parece reflejar un conflicto entre el uso de su L1 (lengua materna, en este caso español) y el uso en inglés. 
El manejo de los pronombres personales puede ser un tanto difícil para los estudiantes de nivel básico A2, agregó la maestra. Así mismo aclaró que en donde más ha notado que existe confusión es en el intercambio en uso de los "subject pronouns" con los "object pronouns" y los "possessive adjectives" con los "possessive pronouns." Algunos ejemplos serian "I don't want to talk to she" en vez de decir "I don't want to talk to her" o "that pencil is my" en vez de "that pencil is mine".

En cuanto al uso de sustantivos en el idioma inglés la maestra expresó que la mayor dificultad está en diferenciar entre lo que son contables y los no contables. Por ejemplo, los estudnates dicen "I bought two breads," o "she bought a water." "I want a candy" en vez de "I want some candy." En forma muy similar a como lo dirían en español. En adición a lo antes mencionado dijo que no hay un patrón claro en la colocación de los adjetivos. En frases sencillas, como "the red car" el carro rojo lo colocan después del sustantivo "the car red," en caso de frases más complejas, especialmente donde hay más de un adjetivo. Por ejemplo "It was a long, beautiful summer" es posible que escriban, "It was a beautiful summer long" o algo por el estilo.

Otro aspecto importante que la maestra destacó durante la entrevista fue las dificultades que presentan los estudiantes en cuanto a la diferenciación del género y expresar cantidades en inglés. La maestra explicó que ha notado que para los estudiantes es fácil adaptarse a no usar género en inglés, ya que a diferencia del español no todos los sustantivos lo poseen. Sin embargo, para ellos resulta un poco confuso que algunos sustantivos como "man" estén contenido dentro del sustantivo de género opuesto "woman" debido a que en español no es igual.

En esa misma tesitura, expresó que a los estudiantes les resulta fácil expresar el plural, excepto cuando se trata del uso de plurales irregulares. Por ejemplo "one foot" singular y "two feet" plural. Lo que normalmente ocurre es que ellos generalizan las reglas de los plurales regulares a los plurales irregulares. En este caso algunos estudiantes dirían "two tooths" en luagar de "two teeth" o dirían "two foots, two feets" en vez de "two feet." Tambien dirían "five fishes" en lugar de "five fish" o dirían "three childs, three childrens" en vez de "three children." A ellos les toma mucho tiempo adaptarse a esa diferencia gramatical entre el inglés y el español.

Cuando se le preguntó a la maestra que dificultades presentan sus estudiantes en la formación de preguntas, orden de las palabras, uso de complemento en inglés y el uso de los adverbios, ella expresó que se muestra que a este nivel muchos estudiantes presentan confusión en agregar el auxiliar do para hacer preguntas cerraras o abiertas. El caso más común es que no utilicen (do) al hacer una pregunta cerrada yes/no question. Por ejemplo, los estudiantes dicen “you want to play?” En lugar de “do you want to play?” o "you like ice-cream?” En vez de “do you like ice-cream?" En el caso de las preguntas abiertas (why-questions) he notado que dicen 
"what you want to do now?" En lugar de "what do you want to do now?" o "where you live?" En vez de "where do you live?" Asi mismo, en notado que los estudiantes no intercambian las posiciones del verbo y del pronombre correspondiente al formar preguntas cerradas usando can. Por ejemplo, los estudiantes dicen "you can play soccer?” en vez de "can you play soccer?”

La maestra explicó que, en lo concerniente al orden de las palabras, la mayor dificultad sucede cuando los estudiantes utilizan los adverbios de frecuencia. Por ejemplo he notado que dicen "I read very often books" en vez de "I often read books." "teacher, they always are late" en vez de "teacher, they are always late" o "we were in plaza el libano shopping clothes" en vez de "we were shopping for clothes at plaza el libano." En ese aspecto, he notado que los estudiantes confunden los adjetivos con adverbios, intercambiando su uso. Por ejemplo, los estudiantes dirían "she speaks English very beautiful" en lugar de "she speaks English beautifuly" o "I feel good" en vez de "I feel well".

Cuando se le preguntó a la maestra que dificultad ella había notado en el uso del complemento por parte de sus estudiantes, ella respondió que el uso del complemento no aplica para el programa del nivel A2. Después de esto la maestra continúo explicando que es un poco difícil para los estudiantes manejar la conjugación de los verbos en inglés, y agregó que ha escuchado algunos casos de uso de palabras inventadas y de falso cognados durante el desarrollo de actividades orales en inglés. En este aspecto, la maestra expresó que los tiempos en pasado y las conjugaciones de los verbos irregulares son muy complejas, requieren de memorización y de mucha repetición. Por otro lado, las reglas para la tercera persona, en el nivel A2, pueden ser un desafío, en niveles más avanzado se pueden ver otras conjugaciones complicadas y es de esperarse que los estudiantes tengan dificultades con ellas.

En adición a lo antes mencionado, la maestra explicó que cada estudiante tiene una combinación diferente de desafíos. Sin embargo, para fines de este trabajo, mencionó tres de los errores que pueden encontrarse, sin intensión de que sea una respuesta definitiva: la conjugación de los verbos irregulares, por ejemplo: en vez de "she went to the park" los estudiantes podrían decir "she goed to the park". Otro error común es la conjugación en tercera persona, especialmente el uso del pasado en la forma interrogativa, y en la tercera persona. Por ejemplo: en vez de "did you play soccer?" puede aparecer "do you played socer?" o en el uso de la tercera persona “does she playes soccer?" entre otros errores de este tipo. También expresó que durante el desarrollo de actividades orales ha escuchado algunos estudiantes decir frases como "not molest me" tratando de decir "don't bother me"; "he is bomber" en lugar de decir "he is a firefighter" o "teacher, I not approved the exam?" tratando de decir "Teacher, didn't I pass the exam?". 
Finalmente, la maestra expresó su opinión que el uso que hacen los estudiantes del nivel A2 de las preposiciones básica (in), (on) y (at), las conjunciones, y la negación en inglés. La maestra expresó que las preposiciones, todas, son sumamente difícil para nuestros estudiantes. Son muchas, no tienen un solo uso y no son iguales a sus usos en español. Por ejemplo, a veces dicen "I am at Santo Domingo" en vez de "I am in Santo Domingo". En lo que tiene que ver con las conjunciones, en este nivel solo trabajamos con tres conjunciones básicas: (and), (but), y (so). No he notado que los estudiantes presenten ninguna dificultad para (and) y (but), pero el uso (so) puede ser un poco difícil de comprender para ellos puesto que esta partícula tiene varios usos en inglés, aunque como conjunción solo sirva para conectar dos clausulas.

Muchos estudiantes cuyo L1 es el español, muestran desafíos con la negación. Por ejemplo, en vez de utilizar (not) más el auxiliar (do) utilizan (no), por ejemplo "I no like..." en vez de “I don't like..." Las negaciones en el pasado con los auxiliares son todo un reto, dijo la maestra, agregando que "al responder estas preguntas me doy cuenta de que hay muchos desafíos que los estudiantes presentan, muchas veces porque siguen pensando en su L1, lo que es de esperarse, pero también es un desafío de poder ayudarles a aprender inglés de una manera en que pueda construir sobre lo que ya saben de su L1 sin que se convierta en un obstáculo.”

\section{DISCUSIÓN}

El análisis de las composiciones indica que el español (L1) influye de manera negativa en el aprendizaje de inglés debido a la dependencia de los estudiantes de estructuras gramaticales de su lengua materna y al uso indiscriminado de la traducción literal desde español al inglés para comunicarse. Las reglas gramaticales no compartibles existentes en ambos idiomas ocasionan una serie de errores sintácticos que se muestran en la forma que los estudiantes organizan sus ideas en la lengua objeto (Cabrera, et al. 2014; Eddin y Hamza, 2019; Filade, Oyinloye, Anwanane, y Oluwabunm, 2019).

Los resultados del preexamen A1 y el examen del nivel A2, analizados mediante la prueba t-student, muestran que el coeficiente de Pearson es menor que el grado de significancia; en donde, el p-valor es menor de 0.05, $(\mathrm{P}(\mathrm{T}<=\mathrm{t})=0.01766<0.05)$. Esto evidencia que los estudiantes que no estan acorde con el nivel del curso incursionan en errores interlinguales, intralenguales y de desarrollo que reflejan la aplicación inadecuada de los conocimientos gramaticales de su lengua materna en el segundo idioma y la poca compresión de la gramática del segundo idioma Richards (1974 citado en Chen, 2016). De igual forma, estos resultados ponen en evidencia que, para componer textos coherentes en la segunda lengua, los estudiantes ne- 
cesitan adquirir ciertas competencias lingüísticas (Tiryakioglu, Peters y Verschaffel, 2019) que les permitan comprender la similitudes y diferencias entre ambos idiomas y asi evitar que la gramática y el vocabulario de la lengua materna influyan negativamente en el aprendizaje de inglés (Khoshsima y Banaruee, 2017).

La encuesta hecha a los estudiantes muestra que un $72 \%$ de ellos refuerza fuera del aula los contenidos aprendidos todos los días o tres veces a la semana, lo cual es un porcentaje alto de reforzamiento de los contenidos, un $21 \%$ lo hace una vez a la semana y un caso a típico $7 \%$ nunca refuerza fuera de clases los contenidos aprendidos en el aula. Ver tabla 1 y figura 2. Estos datos muestran que la influencia negativa de la lengua materna encontrada en la prueba de escritura de los estudiantes no está basada fundamentalmente en su frecuencia de estudio, sino que se deben revisar las técnicas de estudios que ellos están aplicando, lo cual puede ser considerado para futuras investigaciones.

La interpretación de la entrevista en profundidad hecha a la maestra indica que la gramática similar entre el español y el inglés crea cierta confusión de uso en los estudiantes. Esto corrobora los hallazgos de Lockiewicz y Jaskulska (2017) y Samingan (2016) sobre el hecho de que las similitudes lingüísticas entre la lengua materna y la segunda lengua pueden facilitar o dificultar el aprendizaje de la lengua objeto, en otras palabras, pueden resultar en transferencia positiva y/o negativa.

\section{CONCLUSIONES}

Finalmente, es imposible evitar que durante la fase inicial del aprendizaje de una lengua extranjera ocurra una influencia sintáctica desde la lengua materna hacia la segunda. Sin embargo, para minimizar su impacto los estudiantes necesitan desarrollar un alto nivel de comprensión de las conexiones que existen entre forma, significado y uso en la segunda lengua. Para lograr esto, los estudiantes deben manejar con facilidad los contenidos tratados en el aula. En ese sentido, es importante reforzar las áreas más débiles antes de trabajar los temas propios del curso que inicia, y además crear conciencia en los estudiantes sobre los errores que cometen en el L2, y proporcionarles herramientas adecuadas para corregirlos.

Con esta idea en mente, se recomienda la implementación de los enfoques PPP (Present, Practice, Produce) y TBL (Task-Based Learning) en las clases. Estos enfoques permiten presentar los contenidos en la segunda lengua de una forma más digerible para los estudiantes promoviendo el aprendizaje basado en tareas interactivas fuera y dentro del aula (Hafid y 
Nurpahmi 2019; Liu y Yao 2019). Así mismo, el flipped classroom promueve la realización de asignaciones fuera del aula; ocupando el tiempo de clases y la experiencia del docente para potenciar otros procesos de adquisición de la segunda lengua y la práctica de conocimientos (Awidi \& Paynter 2019).

\section{REFERENCIAS BIBLIOGRÁFICAS}

Abbas, N. F., Younus, L. L., \& Khalil, H. H. (2019). Fossilized Use of Active and Passive Simple Present by Iraqi MA Students. Arab World English fournal (AWEF), 10(1), 16-30. Recuperado de http://r. issu.edu.do/l.php?l=231KoQ

Albirini, A., \& Benmamoun, E. (2014). Aspects of second language transfer in the oral production of Egyptian and Palestinian heritage speakers. International fournal of Bilingualism, 18(3), 244273. Recuperado de http://dx.doi.org/10.1177/1367006912441729.

Al-Khresheh, M. H. (2015). A review study of interlanguage theory. International fournal of Applied Linguistics and English Literature, 4(3), 123-131. Recuperado de http://r.issu.edu.do/l.php?l=232p14

Awidi, I. T., \& Paynter, M. (2019). The impact of a flipped classroom approach on student learning experience. Computers \& Education, 128, 269-283. Recuperado de http://r.issu.edu.do/l. php?l=234eMB

Benati, A. (2017). Interlanguage gramar. In J. I. Liontas (Ed.), The TESOL Encyclopedia of English Language Teaching. New York: Wiley. Recuperado de https://onlinelibrary.wiley.com/doi/ abs/10.1002/9781118784235.eelt0077

Brown, H. D. (2014). Principles of language learning and teaching. New Jersey: Prentice Hall Inc.

Burgos, E. G., \& Molina, S. S. (2020). La actitud de estudiantes universitarios hacia Abd-Alrahman, H. A. A. (2017). The Effects of Morph Syntactic Knowledge on English Foreign Learners Translating Abilities (Case study of fourth year students) (Doctoral dissertation, Sudan University of Science \& Technology). Recuperado de http://repository.sustech.edu/handle/123456789/19673

Cabrera, P. A., Gonzalez, P. F., Ochoa, C. A., Quinonez, A. L., Castillo, L. M., Solano, L. M., \& Arias, M. O. (2014). Spanish Interference in EFL writing skills: A case of Ecuadorian Senior High Schools. English Language Teaching, 7(7), p.40-48. Recuperado de https://eric.ed.gov/?id=EJ1076023

Cervatiuc, A. (2019). Analyzing and Understanding Interlanguage. The TESOL Encyclopedia of English Language Teaching, 1-12. Recuperado de https://onlinelibrary.wiley.com/doi/ abs/10.1002/9781118784235.eelt0638 
Chen, J. J. (2016). The development of an interlanguage. Tesol fournal, 3(1), 48. Recuperado de http:// journal.nystesol.org/january2016/NYSTESOLJ-jan2016-47-56.pdf

Choi, K. Y. (2017). Some methodological guidelines for contrastive analysis and error analysis. Fournal of Modern Languages, 10(1), 86-99. Recuperado de https://jml.um.edu.my/index.php/JML/ article/view/3839

Eddin, F. C. K., \& Hamza, N. (2019). The Influence of Mother Tongue on EFL learners' Written Productions The Case of Third Year EFL Students at Mohammed Boudiaf. M'sila University (Doctoral dissertation). Recuperado de: http://dspace.univ-msila.dz:8080/xmlui/handle/123456789/15163

Fauziati, E., \& Maftuhin, M. (2016). Interlanguage Verb Tense Systems of Indonesian EFL Learners. Journal of Foreign Languages, Cultures and Civilization, 4(2), 72-82. Recuperado de http://r.issu. edu.do/l.php?l=241dbz

Filade, B. A., Oyinloye, C. A., Anwanane, B. B., \& Oluwabunmi, O. P. (2019). Mother-tongue influence interference in the study of English language among senior secondary schools in Ago Iwoye, Ogun State. Gender and Behaviour, 17(2), 13007-13015. Recuperado de https://journals.co.za/ content/journal/10520/EJC-16f0e37dec

Filipovic, L. (2018). Speaking in a second language but thinking in the first language: Languagespecific effects on memory for causation events in English and Spanish. International fournal of Bilingualism. 22(2), p. 180-198. Recuperado de https://journals.sagepub.com/doi/ full/10.1177/1367006916661636

Guion, S., Flege, J., Liu, S., y Yeni-Komshian, G. (2000). Age of learning effects on the duration of sentences produced in a second language. Applied Psycholinguistics, 21(2), 205-228. Recuperado de https://doi.org/10.1017/S0142716400002034

Hafid, E., \& Nurpahmi, S. (2019). The PPP Model to Teaching Grammar: Evidence from Indonesian Contexts of the Effectiveness of Explicit Teaching Instructions. The Asian EFL fournal, 23(3.4), 415-421. Recuperado de http://repositori.uin-alauddin.ac.id/15176/

Halcomb, E. \& Hickman, L. (2015). Mixed methods research. Nursing Standard: promoting excellence in nursing care, 29 (32), 41-47. Recuperado de https://pdfs.semanticscholar.org/80b5/037ba5b808d a110334bb60968b83d664138d.pdf

Han, Z. (2012). Fossilization. Recuperado de https://doi.org/10.1002/9781405198431.wbeal0436

Han, Z., \& Tarone, E. (Eds.). (2014). Interlanguage: Forty years later (Vol. 39). John Benjamins Publishing Company. Recuperado de https://doi.org/10.1075/llt.39

Huang, Y. Q. (2017). The Role of L1 in Chinese College Students' English Learning: A Study of 
Kellerman's Theory of Language Transfer. In 2016 2nd International Conference on Economics, Management Engineering and Education Technology (ICEMEET 2016). Atlantis Press. Recuperado de https://doi.org/10.2991/icemeet-16.2017.154

Kalee, S., Rasyid, Y., \& Muliastuti, L. (2018). Error Analysis on the Use of Affixation in Indonesian Paper Written by Thai Student. Lingua Cultura, 12(3), 289-293. Recuperado de https://journal. binus.ac.id/index.php/Lingua/article/view/4307

Kelley, M. F., Roe, M., Blanchard, J., \& Atwill, K. (2015). The influence of Spanish vocabulary and phonemic awareness on beginning English reading development: A three-year (K-2nd) longitudinal study. Journal of Research in Childhood Education, 29(1), 42-59.

Khansir, A. A. (2012). Error Analysis and Second Language Acquisition [Análisis de errores y adquisición de un segundo idioma]. Theory \& Practice in Language Studies, 2(5). Recuperado de http:// www.academypublication.com/issues/past/tpls/vol02/05/22.pdf

Khoshsima, H., \& Banaruee, H. (2017). L1 interfering and L2 developmental writing errors among Iranian EFL learners. European fournal of English Language Teaching. Recuperado de https:// oapub.org/edu/index.php/ejel/article/view/790

Kormos, J. (2020). Specific learning difficulties in second language learning and teaching. Language Teaching, 53(2), 129-143. Doi: 10.1017/S0261444819000442

Lallier, M., \& Carreiras, M. (2018). Cross-linguistic transfer in bilinguals reading in two alphabetic orthographies: The grain size accommodation hypothesis. Psychonomic Bulletin \& Review, 25(1), 386-401. Recuperado de https://link.springer.com/article/10.3758/s13423-017-1273-0

Lao, Y. A. D. (2017). First language interferences into English writing skill of the XIIth grade students of SMA Negeri 1 Kupang in academic year 2017/2018. International fournal of Research-Granthaalayah, 5(12), 33-44. Recuperado de https://www.yandreslao.com/wp content/uploads/2019/07/ Yandres-A.-Dj.-Lao-1.pdf

Larsen-Freeman, D. (2014). Another step to be taken: Rethinking the endpoint of the interlanguage continuum. Interlanguage: Forty years later, 203-220.

Larsen-Freeman, D., \& Anderson, M. (2011). Techniques and principles in language teaching. New York: Oxford University Press

Li, X. (2019). A Probe into the Influence of Language Transfer on English Learning and Its Countermeasures. Recuperado de https://webofproceedings.org/proceedings_series/ESSP/ETMHS\%20 2019/ETMHS19057.pdf 
Liu, X., \& Yao, T. (2019). The Cultivation of College Students' Critical Thinking Ability Based on Taskbased Cooperative Writing. Fournal of Language Teaching and Research, 10(3), 557-568. Recuperado de http://www.academypublication.com/ojs/index.php/jltr/article/view/jltr1003557568

Lockiewicz, M., \& Jaskulska, M. (2017). Polish as L1, English as L2: the linguistic transfer impact on Second Language Acquisition stemming from the interlingual differences: implications for young learners' education. Problemy Wczesnej Edukacji, 37(2), 68-76. Recuperado de https:// www.ceeol.com/search/article-detail?id=583975

Matias Roca, F. M. (2019). The Influence of Catalan and Spanish as L1s in the Learning of English as L2. Recuperado de http://dspace.uib.es/xmlui/handle/11201/150276

Mahmood, A. H., \& Murad, I. M. A. (2018). Approaching the Language of the Second Language Learner: Interlanguage and the Models Before. English Language Teaching, 11(10), 95-108. Recuperado de https://eric.ed.gov/?id=EJ1191679

Moeller, A. K., \& Catalano, T. (2015). Foreign language teaching and learning. Recuperado de http:// dx.doi.org/10.1016/B978-0-08-097086-8.92082-8

Mohammad, K., \& Taie, M. (2016). BICS and CALP: Implications for SLA (Vol. 7). Recuperado de https:// doi.org/10.17507/jltr.0702.19

Mueller, J. T. (2018). English as a lingua franca at the multilingual university. Foreign Language Education in Multilingual Classrooms, 7, 359. Rcuperado de https://doi.org/10.1075/hsld.7.16mue

Relyea, J. E., \& Amendum, S. J. (2019). English reading growth in Spanishspeaking bilingual students: Moderating effect of English proficiency on crosslinguistic influence. Child Development.

Rodríguez, C. T. (2018). The influence of the Spanish language in the learning of English for secondary education students. Entre Línguas, 4(2), 251-267. Recuperado de https://dialnet.unirioja.es/servlet/articulo?codigo $=6732353$

Samingan, A. (2016). First Language Interference in EFL students' composition of IAIN SALATIGA. (Doctoral dissertation, Muhammadiyah University of Surakarta).

Sharma, D., y Tripathi, P. (2018). Error Analysis for English Language Teaching at Primary Level. IfELLH (International fournal of English Language, Literature in Humanities), 6(9), 7. Recuperado de https://www.researchgate.net/publication/331833838 Error_Analysis for_English Language Teaching at Primary Level

Shum, K. K. M., Ho, C. S. H., Siegel, L. S., \& Au, T. K. F. (2016). First language longitudinal predictors of second language literacy in young L2 learners. Reading Research Quarterly, 51(3), 323-344. Recuperado de https://ila.onlinelibrary.wiley.com/doi/abs/10.1002/rrq.139 
Singh, C. K. S., Singh, A. K. J., Razak, N. Q. A., \& Ravinthar, T. (2017). Grammar Errors Made by ESL Tertiary Students in Writing. English Language Teaching, 10(5), 16-27. Recuperado de https:// files.eric.ed.gov/fulltext/EJ1137462.pdf

Steels, L. (2017). Human language is a culturally evolving system. Psychonomic Bulletin \& Review, 24(1), 190-193. Recuperado de https://doi.org/10.3758/s13423-016-1086-6

Tarone, E. (2006). Interlanguage. Dalam K. Brown (ed.) Encyclopedia of language and linguistics. (2rd ed.) Oxford: Elsevier, 747752.

Techo, V. (2016). Research Methods-Quantitative, Qualitative, and Mixed methods. Recuperado de https://doi.org/10.13140/RG.2.1.1262.4886

Tiryakioglu, G., Peters, E., \& Verschaffel, L. (2019). The effect of L2 proficiency level on composing processes of EFL learners: Data from keystroke loggings, think alouds and questionnaires. In Observing Writing (pp. 212-235). BRILL. Recuperado de https://brill.com/view/book/edcoll/9789004392526/BP000010.xml

VanPatten, B., Keating, G. D., \& Wulff, S. (Eds.). (2020). Theories in second language acquisition: An introduction. Routledge.

Yang, Q., \& Xu, Y. (2019). English Teaching Reform in Local Undergraduate Colleges Based on Interlanguage Fossilization. Theory and Practice in Language Studies, 9(3), 313-318. Recuperado de http://www.academypublication.com/ojs/index.php/tpls/article/view/tpls0903313318

Zaman, B., Qi, F., Rafique, R., Fazal, N., Asif, M., \& Shan, L. (2019). Problematic English Vowel Phonemes for Gojri Learners of English. Recuperado de http://www.savap.org.pk/journals/ARInt./Vol.10 (2)/ARInt. 2019(10.2-09).pdf 